\section{Legitimidad territorial de las universidades chilenas: discusiones y desafíos para la vinculación con el medio}

Christian Andrés Quinteros Flores

Universidad Tecnológica de Chile INACAP, Chile.

cquinteros@inacap.cl
Cultura(s) en clave de extensión universitaria Desafíos de gestión
RECEPCIÓN: 03/03/19

ACEPTACIÓN FINAL: 24/05/19

\section{Resumen}

La creciente necesidad de intensificar y robustecer, tanto desde la academia como desde los agentes de planificación regional, los sistemas de extensión universitaria o de vinculación con el medio, hace que nos preguntemos sobre el tipo de exigencias que las políticas públicas aplican hoy a las universidades en Chile respecto de sus modalidades de relacionamiento social y de los enfoques conceptuales y metodológicos que estas tienen en la temática. El presente trabajo se despliega con la premisa de que para los actuales paradigmas de gobernanza resulta necesaria la imbricación de cualquier universidad en las características propias de un territorio incluso desarrollando algún tipo de licencia territorial, si es que pretende genuinamente aportar en procesos robustos de desarrollo. El artículo revisa, en primer lugar, el modelo territorial del sistema de educación en Chile, las actuales políticas y metodologías para la medición de vinculación con el medio, el tratamiento de lo "territorial" desde las universidades, el abordaje de ciertas discusiones sobre legitimidad territorial y el establecimiento final de una propuesta de licenciamiento territorial a las universidades como garantía de sustentabilidad.

Palabras clave: globalización, legitimidad territorial, vinculación con el medio, licencia territorial, gobernanza.
Territorial legitimacy of Chilean universities: discussions and challenges for linking with the environment

\section{Abstract}

The growing need to intensify and strengthen both academia and regional planning agents, university extension systems (E) or linkage with the environment (VcM), makes us wonder about the type of requirements that public policies apply today to universities in Chile regarding their social relations and the conceptual and methodological approaches they have in the subject. The present work is deployed on the premise that for the current paradigms of governance it is necessary the imbrication of any university in the characteristics of a territory even developing some type of territorial license, if it genuinely aims to contribute in robust development processes. The article first reviews the territorial model of the education system in Chile, the current policies and methodologies for the measurement of links with the environment, the treatment of the "territorial" from the universities and finally the approach of certain discussions on territorial legitimacy and the final establishment of a proposal for territorial licensing of universities as a guarantee of sustainability.

Keywords: globalization, legitimacy territorial, linkage with the environment, territorial license, governance.
Legitimida de territorial das universidades chilenas: discussões e desafios para a vinculação com o meio ambiente

\section{Resumo}

A crescente necessidade de intensificar $e$ reforçar tanto da academia e dos agentes do ordenamento do território, extensão sistemas de universidade ou conexão, nos faz pensar sobre o tipo de exigências que a política pública Candidate-se hoje às universidades chilenas em relação às suas relações sociais e às abordagens conceituais e metodológicas que elas têm sobre o assunto. Este trabalho é exibido na premissa de que os atuais paradigmas de entrelaçamento governança de qualquer universidade nas características próprias território ainda em desenvolvimento algum tipo de licença territorial é necessário se for para contribuir realmente para processos de desenvolvimento robustos. O documento analisa primeiro o modelo territorial do sistema de ensino no Chile, políticas e metodologias atuais para medir a ligação, o tratamento da "territorial" de universidades e, finalmente, abordar certas discussões sobre a legitimidade territorial e o estabelecimento definitivo de uma proposta de licenciamento territorial das universidades como garantia de sustentabilidade.

Palabras chave: globalização, legitimidade, articulação territorial com o meio ambiente, licença territorial, governança.

Para citación de este artículo: Quinteros Flores, C. (2019). Legitimidad territorial de las universidades chilenas: discusiones y desafíos para la vinculación con el medio.+E: Revista de Extensión Universitaria, 9(10), 38-59. doi: 10.14409/extension.v9i10.Ene-Jun.8288. 


\section{Introducción}

El modelo neoliberal instalado en Chile en los últimos 40 años ha permeado todos los ámbitos de la vida social del país, fundamentalmente en el ámbito económico, pero de manera extendida también a los ámbitos cultural, político y social. La educación no quedó ajena a esta experiencia y abrió espacio a la liberalización progresiva de sus servicios. Así, la educación superior evidenció una intensa oferta privada en escenarios absolutamente desregulados, lo que ha llevado en la actualidad a presenciar serios problemas de gobernanza educativa. Además, ha permitido hasta la fecha la existencia de universidades e instituciones de educación superior sin ninguna sostenibilidad. Este elemento ha caracterizado el escenario educativo en el desarrollo de las políticas públicas en esta materia. La desregulación ha posibilitado la aparición de distintas instituciones en nuevos territorios aprovechando la masificación de la educación superior.

En este sentido, la preocupación por la dimensión territorial de los fenómenos es, en términos generales, un paradigma moderno en las ciencias sociales y humanas que ha cobrado fuerza inusitada en el ámbito institucional de lo público. En el caso de este artículo, esa preocupación se aplica en la comprensión de las universidades como potenciales líderes del desarrollo territorial que pueden o no hacerse cargo del impacto no solo de sus actividades donde se emplazan geográficamente sino también de las necesidades de la propia comunidad. Esta voluntariedad en el compromiso se explica porque nada las obliga a conocer previamente las características antropológicas, socioculturales o simbólicas de ese territorio, aun cuando las políticas de vinculación con el medio emanadas desde el Estado exijan creciente y recientemente cierta rendición de cuentas en este tema.

En el caso chileno, y a nuestro juicio, estas políticas resultan imprecisas conceptual y metodológicamente, e incentivan a las universidades a centrarse en un interés desmedido y fútil por evidenciar a todas luces un compromiso público un tanto difuso respecto del "entorno" o "medio", estableciendo una agenda de trabajo que obedece más bien a la necesidad de cumplir con ciertos estándares de tecnificación de la educación superior (métricas de calidad) que a la genuina sustentabilidad social, política o ambiental del territorio.

Esta última situación, lamentablemente, aleja a la universidad de procesos de construcción colectiva y legítima de un territorio, procesos donde cada vez existe mayor consenso en que este tipo de instituciones educativas forma parte fundamental de los actores responsables de su desarrollo mediante el establecimiento sistemático, gradual, honesto y transparente de diálogos científicos y técnicos, pero también con otros saberes, simbólicos y populares, donde la compenetración con la historia del lugar, con sus héroes y villanos, con sus aspiraciones y con su memoria, son primordiales en esta tarea. Al respecto es interesante lo que plantea Boaventura de Sousa Santos:

"El conocimiento universitario -o sea el conocimiento científico producido en las universidades o instituciones separadas de las universidades pero que comparten el mismo ethos universitario- fue a lo largo del siglo $x x$ un conocimiento predominantemente disciplinar, cuya autonomía impuso un proceso de producción relativamente descontextualizado con relación a las necesidades del mundo cotidiano de las sociedades. Según la lógica de este proceso, son los investigadores quienes determinan los problemas científicos que deben resolverse, definen las relevancias y establecen las metodologías y los ritmos de investigación. Es un conocimiento homogéneo y organizativamente jerárquico 
en la medida en que los agentes que participan en su producción comparten los mismos objetivos de producción de conocimiento, tienen la misma formación, la misma cultura científica y lo hacen según jerarquías organizacionales bien definidas”. (2007:43)

Las comunidades residentes en los territorios son agrupaciones vivas, con dinámicas internas y externas, que aceptan o no en forma simbólica la instalación de una nueva institución, cualquiera sea su naturaleza. En efecto, es la propia comunidad, a través de la entrega de sus saberes ancestrales y populares, la que otorga a la universidad —de nuevo simbólicamente - la licencia social/territorial que la autoriza a colaborar, por ejemplo, en el rescate y conservación de su patrimonio cultural, en la formación de sus habitantes o en el desarrollo de investigaciones que busquen algún bien público.

Es claro que siempre existe un territorio configurado antes de la instalación de alguna universidad, con una cultura e identidad propias que, si no son reconocidas y respetadas por los agentes externos, se atenta gravemente contra el buen desarrollo de cualquier proyecto, más aún si es de carácter universitario. Esta es la premisa que orienta nuestro trabajo. Se propone en este la creación del concepto llamado "licencia territorial", una suerte de autorización simbólica o explícita que entrega la comunidad territorial a la universidad que decida realizar actividades académicas, de investigación o de extensión dentro de los límites o fronteras del territorio en cuestión. ${ }^{1}$ Es un concepto crecientemente utilizado en conflictos socioambientales.

Este escrito comienza con la presentación de los antecedentes del sistema de educación superior en Chile desde un enfoque territorial y señala cómo ha sido considerado este concepto. Posteriormente, se revisa la institucionalidad vigente en materia de vinculación con el medio para, por último, plantear algunas discusiones que pueden ayudar a los tomadores de decisiones frente a nuevos proyectos universitarios o de extensión.

\section{El modelo territorial del sistema de educación en Chile}

Para Llanos Hernández, el territorio es:

"un concepto que adquiere nuevos contenidos en el contexto de la globalización, son relaciones sociales que desbordan las fronteras de la comunidad, de la nación y que se entrelazan con otros procesos que ocurren en el mundo... Los territorios son espacios de una gran tensión social, están penetrados por el sentido progresivo del tiempo lineal, por la rutina de los tiempos cíclicos y por la vivencia del tiempo simultaneo. La llamada flecha del tiempo ya no tiene el sentido unidireccional que poseía en la época de la modernidad, en los territorios la vida social se abre a un abanico de direcciones, de opciones, de salidas a las acciones sociales de los seres humanos, lo cual implica la posibilidad misma de la fragmentación o de una nueva integración de este tipo de espacios". (2010:214)

\footnotetext{
1) Este concepto se aplica fundamentalmente en Chile a los llamados conflictos socioambientales, y se denomina "licencia social". Al respecto, la amenaza más relevante para el sector minero en Chile es precisamente la licencia para operar que ha evolucionado más allá del enfoque acotado a problemas sociales ambientales. También considera aspectos influyentes en la gestión de las compañías, como el rol del gobierno, el medioambiente, la comunidad, los empleados y los consumidores.

Ver: https://www.reporteminero.cl/noticia/noticias/2019/03/licencia-social-para-operar-es-la-mayor-preocupacion-delsector-minero (consultado el 18 de marzo de 2019).
} 
La cita anterior posee la complejidad conceptual con la que debe observarse hoy el territorio y nos permite contrastar con el desarrollo sociohistórico de las universidades en su relación territorial. Para una mejor comprensión del lector respecto de la crisis de la idea de construcción de territorio que en este trabajo se pretende evidenciar, es fundamental abordar cierta historicidad en el análisis de la calidad del vínculo entre universidad y territorio, no solo en Chile sino también en la región, como veremos luego con la Reforma de Córdoba en Argentina en 1918, con la perspectiva de establecer nuevos desafíos y discusiones al actual modelo de acreditación institucional aplicado en Chile.

Históricamente, en la primera mitad del siglo XX, el modelo territorial universitario en Chile estuvo localizado en reparticiones regionales de las casas centrales de las Universidades de Chile y del Estado, en un modelo centralizado y nacionalista del desarrollo. En una publicación de la Universidad de Santiago de Chile, USACH (ex UTE), se señala:

"Así, y no exenta de dificultades, en 1947, durante el gobierno de Gabriel González Videla, se crea la Universidad Técnica del Estado, que congregó en su seno a las principales escuelas politécnicas de Chile. Estas fueron la Escuela de Artes y Oficios y la Escuela de Ingenieros Industriales, en Santiago; la Escuela de Minas de Copiapó, la Escuela de Minas de Antofagasta, la Escuela de Minas de La Serena, la Escuela Industrial de Concepción y la Escuela Industrial de Valdivia. Más tarde, en julio de1948, se agregaron la Escuela Industrial de Temuco y el Instituto Pedagógico Técnico, destinado a formar profesores para las escuelas industriales y de minas, técnicas femeninas y comerciales. En 1963 se crearon, además, sedes en Talca y Punta Arenas". (2010:22)

De acuerdo con el sociólogo Manuel Antonio Garretón, hasta antes de la Reforma Universitaria de 1981 se buscaba desde el Estado generar un proceso nacional de desarrollo regionalmente equilibrado con fuerte énfasis en el desarrollo tecnológico e industrial propio del período de industrialización que el país atravesaba. Con los gobiernos del Frente Popular (1938 a 1952) comenzó una etapa sólida de reformas sociales y crecimiento industrial con políticas destinadas a generar una base de infraestructura para la producción y un sistema de protección social para la clase obrera y media. "Entre 1940 y 1953, la industria creció en un promedio de 7,5 \% al año y llegó a aumentar su participación en el PNB de un 7,9 \% en 1929 a un 23,0 \% en1955" (Garretón, 2009:48).

En este contexto, el criterio utilizado por el Estado chileno para la distribución territorial de sus universidades obedecía al impulso de una estrategia nacional con énfasis absoluto en el desarrollo productivo e industrial, propio de la época de tránsito al esperado desarrollo, bajo la teoría económica de la modernización.

Sin embargo, a causa de los procesos de rápida proliferación de las universidades chilenas vividos en la década de los ' 80 , por un lado, y de la falta de control sobre la calidad y

2) Posteriormente, en 1981, "la sede de Punta Arenas dio origen al Instituto Profesional de la ciudad y más tarde a la Universidad de Magallanes. El Instituto Tecnológico de Puerto Montt se convirtió en el Instituto Profesional de Osorno, y el de Valdivia quedó traspasado al Instituto Profesional de esa ciudad. La sede de Temuco originó a la nueva Universidad de La Frontera, la de Concepción pasó a formar parte de la Universidad del Bío-Bío y la de Talca se integró al Instituto Profesional que daría origen a la Universidad de Talca. En el norte, la sede Copiapó se transformó en el Instituto Profesional de Copiapó y luego en la Universidad de Atacama. Finalmente, la sede de La Serena se convirtió en la Universidad de La Serena y la de Antofagasta en la Universidad de Antofagasta" (USACH:31). 
sustentabilidad de algunas casas de estudios durante los '90, por otro, nació el denominado "mercado de la educación superior" que, según González, desde 1990 comenzó a expandirse aceleradamente a partir del exponencial aumento en la creación de instituciones privadas (con escasa o nula fiscalización estatal), el incremento sustantivo de la matrícula universitaria y, de modo paralelo, la generación de un conjunto de becas, créditos bancarios y otros sistemas de pago que "actualmente tienen a miles de estudiantes chilenos endeudados, cuestión que les significa un compromiso económico que se puede extender por décadas" (2017:112).

En efecto, a partir de esa época se experimentó un proceso de fragmentación del modelo universitario chileno:

"El sistema de educación superior en Chile se atomizó bajo la dictadura militar de 1973 a 1990 especialmente en la década de 1980. En 1981 el gobierno chileno fragmentó las dos universidades estatales de Chile (Universidad de Chile y Universidad Técnica del Estado) las cuales concentraban el $65 \%$ de la matrícula de los estudiantes de educación superior. El gobierno cambió también el financiamiento público directo de las instituciones a aranceles desregulados apoyados por becas y créditos. También permitió la creación de nuevas instituciones privadas de educación superior con escasa o ninguna relación. Surgieron así basadas en criterios privados 40 universidades, 78 IP y $161 \mathrm{CFT}^{3}$ entre 1980 y 1990. Sin embargo, las instituciones acreditadas mantuvieron plena autonomía para crecer, por lo que la expansión del sistema pasó de la incorporación de las nuevas instituciones a la proliferación de sedes bajo un marco institucional”. (OCDE, 2017)

El número de planteles de educación superior alcanzó los 398 en 2013, con mayor concentración en áreas urbanas claves, como el área metropolitana de Santiago, la región de Valparaíso y la región del Bio-Bio. Las universidades privadas no pertenecientes al Consejo de Rectores de las Universidades Chilenas(CRUCH) 4 y los IP han sido especialmente activos en la introducción de nuevas sedes (OCDE, 2017:183).

Siguiendo a González,

"el fortalecimiento de este mercado de la educación superior ha enfrentado las funciones históricas de las universidades, su compromiso con la sociedad y con los sectores más desposeídos, frente a la necesidad de autofinanciamiento, mayor y más progresivo ingreso de estudiantes, venta de servicios, estrategias comerciales, que contradicen la naturaleza filosófica y política que explica la existencia de la educación superior como un potencial de bienestar y desarrollo para los países latinoamericanos". (2017:110)

Hasta este punto, es posible señalar que se advierte en este desarrollo histórico una cierta paradoja de la vinculación con el medio por parte de las nuevas universidades. Con la fragmentación de la Universidad de Chile y dela Universidad Técnica del Estado se in-

3) IP se refiere a los Institutos Profesionales y CFT a los Centros de Formación Técnica. Ambos tipos de instituciones pertenecen al sistema de Educación Superior chileno.

4) El CRUCH se crea en el 14 de agosto del año 1954, en virtud a lo establecido en la Ley $N^{\circ} 11.575$, artículo 36 , letra c, que lo define como un organismo de coordinación de la labor universitaria de la nación. Se le asigna también la tarea de mejorar el rendimiento y calidad de la enseñanza universitaria a través de un trabajo propositivo. Está integrado por los rectores de las 27 universidades estatales y no estatales con vocación pública del país y lo preside el ministro de Educación. 
tentó promover e impulsar el desarrollo regional, aprovechando la estructura dada nacional y centralizadamente con énfasis en lo productivo y extractivo, despojado de cierto criterio territorial-local genuino y más integral, y sin ninguna política estatal que incentivara la preocupación por fortalecer los vínculos de esa universidad con las identidades locales y los saberes populares. El criterio territorial aplicado, entonces, debiera haber considerado los nuevos enfoques de desarrollo, incorporando progresivamente el reconocimiento de las identidades locales, de sus discursos, de sus narrativas, de sus subjetividades, que es hacia donde apunta este trabajo, como veremos luego.

Con posterioridad a la reforma universitaria de 1981, y dadas las políticas de mercado impulsadas por el gobierno militar, creemos que lo único que se logró fue la distorsión de la identidad universitaria o, mejor dicho, de su verdadera misión institucional, cual es la de generar nuevo conocimiento situado que aporte al desarrollo del lugar donde decidió instalarse. A partir de ese momento, cada universidad pública o tradicional, y especialmente las privadas, podían aperturar sedes regionales sin haber tenido algún contacto previo con su territorio. Prevalecía así su identidad "corporativa" más que su identidad "territorial", y menos, como veremos luego, la consideración de sus "paisajes" o "discursos" o los "imaginarios urbanos", en el lenguaje de Lindon, o de saberes populares en el de Boaventura de Sousa Santos. El mercado lo permitía y en cierta medida hoy lo sigue permitiendo, aunque con mayor fiscalización y regulación estatal. Pero algo distinto ocurre hoy en Chile, ya que los nuevos paradigmas de gobernanza y sustentabilidad imperantes en el discurso público, sumados a nuevos repertorios epistemológicos que relevan los saberes populares y decoIonializados, exigen una especificación dela gestión universitaria centrada en la territorialización, con conceptos claros, alcances y límites definidos y diálogos ciudadanos.

Entonces, la fácil reproducción de universidades en el territorio nacional —dejada en manos del mercado y de la oferta/demanda - posicionó en un segundo plano otros asuntos fundamentales como la consideración de la legitimidad de una institución con el "nuevo" territorio escogido y con su compromiso con la comunidad. Incluso, hasta antes de la promulgación de la Ley de Acreditación, esta situación era absolutamente invisibilizada por la política pública, la que en apariencia concebía la política de extensión universitaria como una práctica comunitaria de las distintas manifestaciones culturales y formativas de la universidad. Sobre "cuánto" esa universidad conocía el territorio o tenía una vocación social especial para conducir el desarrollo social y cultural de esa región era un asunto desconocido o al menos invisibilizado. Desde esta perspectiva, el crecimiento del número de universidades de menor calidad y de sus sedes universitarias fue desmedido durante los años 90 y principios del siglo XXI:

"El caso de la Universidad del Mar ilustra como este crecimiento de las sedes podría conducir a serios problemas en un contexto de débil gobernanza institucional y supervisión externa inadecuada. (...) Chile aún enfrenta una dura batalla para desarrollar un sistema de educación superior que merezca la confianza del público y pueda cumplir con su promesa de promover el desarrollo económico, social y cultural”. (OCDE, 2017:184)

Hemos desarrollado hasta aquí un marco socio histórico que permite cierta comprensión de la precariedad y dificultades del modelo universitario en Chile para considerar un concepto complejo de territorio en sus políticas. Desde la perspectiva que queremos abordar en 
este artículo, creemos que el territorio es un espacio disputado antropológica e instrumentalmente por universidades con distintas identidades, creencias e ideologías. De esta manera, universidades confesionales, tecnológicas, laicas, etc., se disputan cada día la legitimidad de su presencia en un territorio. Algunos argumentan que esa preocupación es producto del legado del fundador; otros, que es parte de su misión apostólica, etc. Pero, ¿quién puede validar con total seguridad esta condición?

Una estrategia desde el Estado para validar la legitimidad territorial de las universidades ha sido la dimensión denominada "vinculación con el medio", una construcción conceptual que privilegia la interacción recíproca y el beneficio mutuo en el binomio territorio-universidad, que reemplaza la visión más extensionista o de difusión de la universidad. Al respecto, resulta interesante considerar lo propuesto por González:

"Aparece así un nuevo reemplazante que toma su lugar, sin resistencia, en la cultura conceptual y discursiva de las universidades chilenas: la 'vinculación con el medio', que deshistoriza y anula la memoria social y política de una institucionalidad con un fuerte componente simbólico para el país que tuvo sus gérmenes en 1843, año en que se fundó la gran universidad nacional: la Universidad de Chile. Este cambio de denominación resultó ser un nuevo travestismo conceptual, característico de gestiones políticas que se precian de técnicas planificadas y eficientes pero que ocultan su fin último: ir fragmentando los avances en procesos que contienen naturalmente un fuerte impacto social ideológico, como todo lo relativo al trabajo territorial, la relación con los actores sociales o la relativización del verdadero resultado que tienen las disciplinas a la hora de pensar las comunidades, las regiones, las provincias, los países y, finalmente, el mundo". (2017:112)

De este modo, en Chile, las políticas públicas en la denominada "tercera misión" de las universidades han transitado desde un enfoque de extensión a un enfoque de bidireccionalidad de la acción universitaria en el territorio. En efecto, desde este punto de vista, la comunidad y el territorio no son más sujetos pasivos en el mundo académico, por el contrario, determinan estratégicamente su desarrollo. Dependiendo del diagnóstico territorial-local, una universidad decide qué especialistas formar, qué capital humano promover, qué estrategia de desarrollo proponer, entre otras muchas cuestiones, y no solo resuelve la pregunta reduccionista sobre qué necesita el mercado productivo, como ocurría a mediados del siglo XX. En esta línea de discusión, es claro que el concepto de territorio imperante en la década del ' 50 en Chile, y el de territorio que actualmente se utiliza en círculos académicos han cambiado de forma categórica. La incorporación del paisaje, de su simbología, de los discursos de los sujetos, hasta de sus emocionalidades involucradas en su construcción, son aspectos que hoy deben ser atendidos por los tomadores de decisiones en materia de educación superior en Chile.

Al respecto y profundizando en este análisis, es conveniente referirse a los nuevos enfoques de territorio que priman en las ciencias sociales y que han dado paso progresivamente a enfoques centrados en el desarrollo de discusiones simbólicas de lo que ocurre en un espacio determinado. Efectivamente, el concepto de territorio ha transitado desde enfoques más objetivos y concentrados en la materialidad hacia otros más subjetivos y constructivistas que hacen alusión a percepciones, costumbres, creencias de los actores que en ellos viven, lo que Alicia Lindon denomina los "imaginarios sociales". Por otra parte, la preocu- 
pación por el territorio ha pasado desde ser un enfoque disciplinar a representar un área de interés multi e interdisciplinar. Ya lo señala Llanos Hernández:

"El territorio al interior de la geografía constituyó un concepto disciplinario, este mismo sentido se presenta cuando otras disciplinas lo incorporaron a su campo de estudio; sin embargo, en la actualidad el territorio es más que un concepto disciplinario, pues ha pasado a convertirse en un concepto interdisciplinario y a formar parte de los referentes teóricos de las diversas disciplinas que tienen como objeto de estudio los múltiples tipos de relaciones que despliegan los seres humanos". (2010:213)

Para este autor, la noción de región es ya insuficiente para conocer e interpretar la nueva realidad de los seres humanos y la naturaleza. El territorio es un concepto teórico y un objeto empírico más flexible; no solo continúa representando el soporte geopolítico de los Estados nacionales, sino que es una manifestación más versátil del espacio social como reproductor de las acciones de los actores sociales. Constituye, para este autor, un objeto que puede ser analizado desde la perspectiva interdisciplinaria, que ha pasado del reduccionismo fisiográfico a un concepto que existe porque culturalmente hay una representación de él, porque socialmente hay una espacialización y un entramado de relaciones que lo sustentan, y porque política y económicamente es una de las herramientas conceptuales más fuertes en la demarcación del poder y del intercambio (Llanos Hernández, 2010).

Para Alicia Lindon (2007), el concepto de geografía humana parece ofrecer una puerta particularmente relevante por hallarse a medio camino entre lo material del territorio y lo inmaterial: en esta disciplina existe una fuerte tradición de estudio de la ciudad y lo urbano con todo el peso de la materialidad que ello implica. Al mismo tiempo, en esta disciplina se ha desarrollado una tradición de estudio del espacio como mundo de los sólidos y de los no sólidos (espacio vivido, percibido, representado, experimentado, lugar...). Como mencionamos, es lo que esta autora denomina los "imaginarios urbanos". Para ella, estos constituyen una mirada que da cuenta de la relación entre lo no material, la subjetividad espacial, y la ciudad en cuanto a sus formas materiales y a las prácticas que se inscriben en esas formas materiales. Las prácticas sociales contribuyen a la "hechura" de la ciudad material y, a la vez, adquieren ciertos rasgos a partir de la materialidad de la ciudad.

En este camino hacia la subjetivización del territorio, algunos autores se refieren a la cuestión incluso como la "emocionalización" del paisaje y hablan de su valor contemporáneo, vinculado a una manifiesta mercantilización de la cultura, la construcción comercial de identidades, el triunfo de la inautenticidad, de la representación inducida o la economía del simbolismo, y abren amplias expectativas en el estudio del valor simbólico del paisaje. El acercamiento que experimenta la praxis geográfica al estudio del intangible espacial, relacionado con el descubrimiento de las geografías emocionales, además del creciente interés que dispensan las ciencias de la comunicación al discurso territorial, permite, mediante una propuesta de fusión de los saberes geográfico y comunicativo, plantearse abiertamente el estudio comunicativo del paisaje.

Para Nogué y Vela es cada vez más relevante el papel que desarrolla el territorio como mediador de procesos de comunicación, así como en los procesos de consolidación o creación de identidades locales, en todas las escalas. Ahí es, precisamente, donde el paisaje adquiere un importante protagonismo, porque este es, per se, un aparador cultural y un escenario de 
lenguajes. Si es verdad que en el espacio se encuentra el mensaje y, por lo tanto, el territorio con carga simbólica ejerce la función de transmisor de este mismo mensaje, también lo es que el paisaje adquiere en este proceso un protagonismo indiscutible (Nogué y Vela, 2011).

En síntesis, la noción de territorio que subyace a los planteamientos expresados en este trabajo está referida a aquel "lugar" donde se instala una universidad y que no solamente involucra límites físicos o topográficos, sino discursos, narrativas, actores, lenguajes, relaciones de poder, dinámicas internas, conflictos. Es decir, se trata de un concepto de alta complejidad. Siguiendo a Arzeno:

"En términos teórico-metodológicos, considerar la complejidad analítica del concepto de territorio nos puede ayudar a poner el foco en aspectos relevantes de la realidad que estudiamos/intervenimos, tales como las relaciones de poder que estructuran los espacios (tanto en sus aspectos materiales como en cuanto a las representaciones dominantes, a los aspectos más simbólicos, etc.)". (2018:10)

\section{El tratamiento de lo "territorial" desde las universidades}

Una probable explicación al distanciamiento actual de las universidades con la dimensión simbólico-territorial del impacto de sus actividades es la escasa conversación de sus curriculum o disciplinas con ella. Más bien, esta dimensión es un espacio disciplinar hegemonizado por geógrafos, arquitectos o urbanistas, excluyendo a otras áreas del conocimiento. La formación en "territorio" es incipiente en las universidades. Solo analizando el caso de los trabajadores y las trabajadoras sociales en Chile, en un estudio reciente realizado por Quinteros se indica que:

"Pareciera ser que la política pública en Chile avanza a pasos agigantados a la concepción del territorio como un enfoque conceptual y metodológico de intervención social para la focalización de los recursos. De esta manera, la oferta programática de programas de inversión social hoy deja atrás concepciones economicistas del desarrollo local y migran hacia marcos conceptuales que relevan el concepto de territorialidad como factor que delimita este nuevo objeto. Sin embargo, la gestión del territorio ha sido un campo donde disciplinas como la arquitectura o la geografía han ejercido cierto monopolio disciplinar amparado en prácticas históricas e institucionales de larga data y donde la participación de nuevas áreas profesionales es evaluada con cierta cautela". (2018:11)

En este mismo sentido, según Menardi et al., el enfoque territorial aparece escasamente en las carreras y planes de estudios y se lo relega en la formación profesional, lo que incide de manera negativa, ya que "la adopción de la perspectiva territorial contribuye con los aprendizajes vinculados a la pluralidad, particularidades y diferencias de los procesos sociales en el espacio" (2016:79).

Por otra parte, y asociado al punto anterior, existe un serio problema epistemológico y metodológico con los límites del impacto de las acciones universitarias y, en consecuencia, en la definición conceptual de territorio, para algunos llamados indistintamente y con menor rigor "área de influencia", "entorno", "medio externo" o "área de interés" de cierta universidad. Establecer el alcance del concepto resulta fundamental para homogeneizar discursos en esta tarea, más aún cuando el capital cognitivo en la temática está tan centralizado y 
monopolizado por ciertas disciplinas o profesiones. El límite territorial es fundamental en el desarrollo del plan estratégico de tal o cual universidad, definición que permea sus recursos, identidades, patrimonio, relaciones de solidaridad, etc. Por ejemplo, ciertas universidades llamadas "regionales" del $\mathrm{CRUCH}$, ¿tienen una responsabilidad solo en ese ámbito o su impacto se espera también a nivel nacional? Estas universidades regionales generadas después de la reforma a la Universidad Técnica del Estado, ¿han logrado generar un proceso identitario con énfasis en el territorio local o siguen teniendo el sello de universidad nacional con énfasis en lo productivo de la extracción minera?¿Qué criterios consideran entonces estas universidades para definir su concepción territorial?

Hoy por hoy, convergen en los territorios muchas universidades de origen público y privado, todas ellas con declaraciones grandilocuentes sobre sus "significativos" aportes en investigación y en transferencia tecnológica hacia las comunidades. ¿Pero tiene cada una de ellas establecida una pertenencia real a ese territorio? ¿Tienen las universidades la convicción política suficiente para realizar cualquier ${ }^{5}$ actividad allí? ¿Se puede hablar de "derechos" territoriales de unas sobre otras? ¿Prevalece el "derecho" que otorga la institucionalidad histórica?

En tiempos de plena globalización, las instituciones y organizaciones sociales en general deben transitar hábilmente y con ductilidad entre espacios globales y locales justificando en forma permanente su existencia. Realizan este esfuerzo para no perder legitimidad social y se hacen cargo a la vez, de manera responsable, de sus impactos locales. En tanto, la mera referencia al territorio adscrito o adquirido es una condición obligada para toda institución pública o privada, más aún si corresponde a una del área educativa, como es el caso de las universidades, puesto que desde el punto de vista ético se les exige desarrollar un enfoque inclusivo y colaborador con las comunidades de donde decida emplazarse geográficamente. En este último tema no hay vuelta atrás. Es así, como para la Comisión Nacional de Acreditación (CNA) cuando una universidad atraviesa por un proceso de acreditación institucional, ya que específicamente debe someterse a la evaluación de la dimensión de vinculación con el medio y evidenciar de modo público sus logros en el marco de los procesos de acreditación institucional. Gran parte de la eficacia de una vinculación con el medio pasa por demostrar frenética y voluminosamente su "aporte" a la comunidad mediante una serie de indicadores establecidos por la CNA.

Como revisamos anteriormente,

“El término 'vinculación con el medio' no llegó aislada e individualmente. Fue producto de toda una renovación discursiva que combinó elementos de la tradición extensionista chilena y latinoamericana (sobrevivientes), con otras conceptualizaciones provenientes del mundo de la empresa y la economía, configurando una nueva Torre de Babel que cada cierto tiempo va renovando, naturalizando y deshistorizando aquellas prácticas y experiencias que tienden a tensionar la estructura jerárquica de la gestión y el sentido más genuino del hacer y pensar universitario". (González, 2017:114)

5) Las cursivas son del autor, utilizadas con el propósito de visibilizar la inexistencia de reglas del juego en el desarrollo de estas organizaciones desde un enfoque institucionalista. Siguiendo a Joan Prats, las instituciones establecen los incentivos y las restricciones que guían la percepción, las preferencias y las decisiones de individuos y organizaciones. 
La CNA es un organismo público, autónomo, cuya función es verificar y promover la calidad de las universidades, institutos profesionales y centros de formación técnica autónomos, y de las carreras y programas que ellos ofrecen. La CNA Chile se enmarca dentro de la ley que establece un Sistema Nacional de Aseguramiento de la Calidad de la Educación Superior, $\mathrm{N}^{\circ}$ 20.129. Dentro de sus funciones están la de desempeñar acreditación institucional, es decir, pronunciarse sobre la acreditación institucional de las universidades, institutos profesionales y centros de formación técnica autónomos, autorizar agencias acreditadoras, y acreditar las carreras de pregrado y programas de posgrado. Específicamente, la acreditación institucional en vinculación con el medio se refiere al conjunto de nexos establecidos con el medio disciplinario, artístico, tecnológico, productivo o profesional, con el fin de mejorar el desempeño de las funciones institucionales, de facilitar el desarrollo académico y profesional de los miembros de la institución y su actualización o perfeccionamiento, o de cumplir con los objetivos institucionales. Para acreditar esta área, las instituciones deben contar con mecanismos sistemáticos de vinculación con el medio que se refieran a una parte sustantiva del quehacer de la institución y que tengan un impacto significativo en su área de influencia.

Pese a la complejidad de la medición anterior, la vinculación con el medio definida por el Estado es efectuada por los organismos evaluadores a través de los siguientes indicadores: a) proyectos de vinculación con el medio vigentes, que considera el nombre del proyecto, los años (desde-hasta) y la descripción de los alcances de los objetivos; b) los convenios vigentes, el nombre del convenio, los años (desde-hasta) y la descripción de los alcances de los objetivos; y c) indicadores disponibles de impacto o cobertura. A la luz de estas exigencias formales, claramente estamos frente a una diferencia abismal respecto de la complejidad entre lo que se pretende medir y lo que realmente se mide.

Como se puede apreciar, no existen desde el Estado chileno, y desde una perspectiva institucionalista, reglas del juego o incentivos que exijan la aplicación homogénea de un cierto enfoque territorial a las actividades de extensión de una universidad, aunque exijan verificar el cumplimiento de esto antes del inicio de sus actividades, situación que se produce cuando un "directorio" de alguna universidad decide ampliar su distribución de oferta en otras localidades manteniendo un enfoque centralista en las decisiones.

Por otra parte, la nueva ley creada por la Subsecretaría de Educación Superior ( $\left.N^{\circ} 21.091\right)$ señala que la educación superior cumple un rol social que tiene como finalidad la generación y desarrollo del conocimiento, sus aplicaciones, el cultivo de las ciencias, la tecnología, las artes y las humanidades, la vinculación con la comunidad a través de la difusión, valorización y transmisión del conocimiento, además del fomento de la cultura en sus diversas manifestaciones, con el objeto de aportar al desarrollo sustentable, al progreso social, cultural, científi$\mathrm{co}$, tecnológico de las regiones, del país y de la comunidad internacional. Asimismo, la educación superior busca "la formación integral y ética de las personas, orientada al desarrollo del pensamiento autónomo y crítico, que las incentive a participar y aportar activamente en los distintos ámbitos de la vida en sociedad de acuerdo con sus diversos talentos, intereses y capacidades" (art. 1).

Específicamente con relación a las universidades, esta ley señala:

"Las universidades son instituciones de educación superior cuya misión es cultivar las ciencias, las humanidades, las artes y las tecnologías, así como también crear, preservar y transmitir conocimiento, y formar graduados y profesionales. Corresponde a las univer- 
sidades contribuir al desarrollo de la cultura y la satisfacción de los intereses y necesidades del país y sus regiones. Éstas cumplen con su misión a través de la realización de docencia, investigación, creación artística, innovación y vinculación con el medio". (art. 3)

En tanto, en el área de la institucionalidad científica, la ley creó recientemente el Ministerio de Ciencia, Tecnología, Conocimiento e Innovación, que se estructura en torno a tres ámbitos: a) ciencia, tecnología e innovación de base científico-tecnológica, y formación de recursos humanos altamente calificados, a cargo del Ministerio de Ciencia, Tecnología, Conocimiento e Innovación y de la Agencia Nacional de Investigación y Desarrollo; b) fomento productivo, emprendimiento e innovación productiva o empresarial, desarrollo tecnológico para fines productivos y fortalecimiento de recursos humanos para este ámbito, a cargo del Ministerio de Economía, Fomento y Turismo y de la Corporación de Fomento de la Producción; y c) formación de técnicos y profesionales y del conocimiento y el cultivo de las ciencias, las artes y las humanidades en las instituciones de educación superior, a cargo del Ministerio de Educación.

Atendiendo las nuevas directrices institucionales, tanto en la nueva institucionalidad de educación superior como en la científica, vemos que no se explicitan abordajes conceptuales sobre territorios que al menos consideren sus concepciones disciplinares o epistemológicas más básicas, por lo que se debería apuntar de modo urgente a la generación de diálogos entre saberes científicos institucionales y saberes propios de la comunidad.

"El impacto más relevante en este discurso es la cohesión integral de un modelo de extensión crítica que entra, de modo emergente, a disputar los saberes legitimados al interior de la universidad con aquellos provenientes de los territorios, por intermedio de la validación política y orgánica de instancias institucionales que formalizan esta experiencia a nivel micro y macro: desde asignaturas pensadas desde y con el territorio; hasta escenarios institucionales que funcionan como traductores en doble sentido; pasando por las horas asignadas a los labores docentes, estudiantiles y administrativas de la vinculación con el medio; y teniendo presente que en ese ejercicio de diseño y liberación los actores estratégicos del territorio también influyen en sus memorias, prácticas y saberes a nivel de pares." (González y Saravia, 2017:145, en González, 2017:120)

A esta altura del trabajo, creemos que estamos en condiciones de plantear algunas discusiones que nos lleven a incorporar la inclusión de este enfoque en las políticas públicas educativas. A continuación, entonces, señalaremos nuevas variables para profundizar en la medición tecnocrática del proceso de vinculación con el medio. La sintonía que ciertos programas académicos tienen con el desarrollo productivo de la zona donde se insertan resulta clave y "básica" al momento de justificar su presencia e inserción territorial.

Según González:

"por eso, el concepto de bidireccionalidad que se repite en el discurso de los actores universitarios chilenos y en los documentos orientadores que han emanado de 'comités de expertos' reduce la importancia del reconocimiento, la caracterización heterogénea por naturaleza y las complejidades identitarias e históricas que son parte constitutiva del proceso de extensión. Es importante, por lo tanto, hablar de procesos dialógicos, heterogéneos y plurales que visibilizan identidades, modos de operación, historicidades, estrategias de producción y resistencia". (2017:116) 
En el caso de las acreditaciones en vinculación con el medio de universidades con vocación regional, esta territorialización de su acción es muchas veces una suerte de teorización o juego conceptual donde cada cierto tiempo se muestra a la autoridad pública el "alto grado de compromiso" que la universidad evaluada tiene con el desarrollo de esa comunidad y, por lo tanto, queda fuera de todo cuestionamiento su presencia beneficiosa para ese lugar. No calificar adecuadamente en esta medición de la acreditación se transforma en el "pecado original" de una institución que deja ver un flanco débil frente a la competencia pública y/o privada en una de las tres misiones universitarias junto a la docencia y a la investigación. No solo tiene impacto en la asignación del número de años de acreditación.

En el caso de las universidades privadas -algunas de ellas deambularon entre proyectos nacionales y regionales - creadas con posterioridad a la reforma del 1981, muchas comenzaron sus operaciones en regiones con estrategias muy fundamentadas de crecimiento y pertinencia local. Una vez obtenida su autonomía y liberadas de la supervisión estatal, revelaron su "agenda oculta" y cambiaron radicalmente su política hacia la expansión de sus operaciones a diferentes regiones (Prieto y Noel, 2008; Brunner, 2009), lo que se transparenta en el hecho de que casi la totalidad de ellas trasladan sus sedes centrales o rectorías a Santiago, fortaleciendo el centralismo del sistema. Son estas instituciones las que expandieron la matrícula en Chile aplicando estrategias de mercado y modelos gerenciales de dirección (Brunner, 2009).

Según un trabajo reciente de Rivera, el análisis del caso de la Universidad de Aysén (universidad pública que inició sus actividades en 2015) permite describir y comprender algunas de las dificultades que muestran las universidades regionales en Chile para vincularse con su territorio e impactar en el desarrollo local. Este problema, que evidencia una arista de desigualdad y debilidad del sistema de educación chileno, es una constante en las diferentes evaluaciones nacionales e internacionales. La tesis de los autores es que estas dificultades son algunas de las tantas expresiones de la persistencia de un clivaje centro-periferia, que se manifiesta en una insensibilidad territorial o paradigma centralista de las políticas públicas en Chile, lo cual, con independencia de la orientación política del gobierno (democrática o autoritaria) o la preeminencia de la planificación estatal o del mercado como regulador del sistema, ha tendido a respuestas homogéneas y centralistas para enfrentar realidades y necesidades territoriales diversas (Rivera et al., 2018).

Ahora, en el marco de la construcción de universidades públicas regionales en Chile nos planteamos la siguiente pregunta:

"¿Es posible la existencia de una diversidad de propósitos que recojan las particularidades territoriales en un sistema universitario altamente centralizado y con estímulos homogéneos? A partir de este propósito, se lleva a cabo un análisis desde una mirada territorial que posibilita explicar cómo las particularidades regionales entran en fricción con las orientaciones políticas del sistema universitario". (Rivera et al.:428)

En efecto, cuestiones como: ¿qué tan comprometida está tal o cual universidad o su sede con la Estrategia Regional de Desarrollo (ERD) o con el Plan Regulador Comunal 
(PRC) de una determinada comuna o su con su Plan de Desarrollo Comunal (PLADECO)? ${ }^{6} \mathrm{O}$ como: ¿los proyectos regionales amenazan los intereses de la sede central o, en su defecto, los compromisos políticos de esta con la autoridad central? Claramente se aprecia un problema de lealtad política territorial que, de no ser la misma debidamente explicitada, puede generar tensiones a nivel corporativo. Ante este contexto, diversas críticas han surgido en las regiones desde una perspectiva territorial y esbozado las limitaciones o efectos negativos del sistema universitario chileno, como centralismo, dependencia y sub desarrollo (Von Baer, 2006).

De esta forma, el caso de la Universidad de Aysén ${ }^{7}$ puede servir para sacar algunas conclusiones preliminares respecto de las limitaciones del sistema universitario chileno desde la óptica territorial.

a) Los estímulos homogéneos generan comportamientos estándares, insensibles a las necesidades locales, que las desvinculan de su entorno inmediato y de las posibilidades de impactar en el desarrollo regional.

b) La competencia por recursos basada en la calidad (mercado) y la ausencia de financiamiento directo estatal (basal) condiciona a todas las universidades a competir por matrícula, la que para territorios aislados y de baja densidad demográfica resulta infranqueable.

c) Tanto universidades como Gobiernos Regionales (GORE) dependen administrativa y financieramente del centro (Santiago), por lo que el diálogo y la coordinación directa entre ambos resultan complejos.

Como se puede apreciar, el problema en realidad es bien intrincado. Tanto las universidades y los órganos descentralizados del Estado como los gobiernos regionales o los municipios definen básicamente su presupuesto con recursos provenientes o resueltos desde el nivel central, los primeros con el FNDR (Fondo Nacional de Desarrollo Regional) y los segundos con la alta contribución que entrega el FCM (Fondo Común Municipal), reproduciendo persistentemente el problema de la inadecuada vinculación con el territorio que tienen algunas universidades nacionales con presencia regional o definitivamente regionales. La diversidad de instituciones es múltiple, pero el problema es básicamente el mismo.

En este sentido, el desarrollo tiene un componente territorial que requiere acuerdos y consensos entre los distintos actores locales, organizaciones públicas y privadas que trabajan en un territorio en procesos de investigación y formación básica, técnica y profesional. Al respecto, es importante revisar lo planteado por autores como Menardi o Sergio Boisier. ${ }^{8}$

6) PLADECO se refiere al Plan de Desarrollo comunal de una municipalidad en Chile, que se confecciona cada 4 años para orientar el desarrollo. Es un instrumento participativo y estratégico. PRC se refiere al plan de ordenamiento territorial de la Municipalidad, de carácter más técnico e inconsulto. Ambos instrumentos de gestión territorial establecidos en la Ley N¹8.695, Ley Orgánica Constitucional de Municipalidades.

7) La Universidad de Aysén fue creada el año 2014 y declara entre sus principios constituyentes su vocación regional: busca potenciar la identidad y el desarrollo regional y, al mismo tiempo, contribuir y dialogar con el mundo, generando capacidades en la región y sus habitantes.

8) Podríamos denominar a este proceso "territorialización del desarrollo". De acuerdo con Menardi et al. y Sergio Boisier, el desarrollo se transforma en un proceso político, pues implica la construcción y definición de acuerdos y desacuerdos, de consensos y conflictos. Como todo proceso, se da en el transcurso del tiempo y es promovido, pensado, planeado o inducido por diversos actores locales que habitan un territorio. Boisier plantea su concepto de capital sinergético del territorio e individualiza una serie de subcapitales, tales como el social, el cultural, el institucional, el cívico, entre otros, todos basados en las lógicas de confianza y de cooperación entre actores. 
Las líneas que siguen pretenden contribuirá las decisiones de los gestores de proyectos universitarios en Chile en cuanto a los elementos que debe considerar una genuina preocupación por el territorio y no solo una propuesta para cumplir con estándares insuficientes de vinculación.

¿Cómo hace una nueva universidad para validarse como un aliado estratégico de las comunidades? ¿Es posible llegar a un tipo de licencia pública entregada por la propia comunidad para poder validar su presencia?

A juicio de este autor, Chile ha iniciado un poco tarde la discusión sobre la relación con el territorio por parte de las universidades, discusión que lleva en otros países de la región ya casi un siglo. En efecto, la Reforma de Córdoba de 1918 en Argentina estableció un precedente en la materia que sin dudas ha fortalecido largamente este tipo de cuestiones en ese país:

"A partir de Córdoba (Reforma de Córdoba de 1918) se inauguran una serie de procesos que buscan transformar los sentidos del trabajo universitario. Entre ellos, el que tiene que ver con la valorización, rescate y visibilización de los saberes situados en la externalidad de los límites convencionales de la universidad. Esto a partir del convencimiento de que la formación del conocimiento científico, favorecido por la universidad convencional, no ha sido capaz o no le ha interesado tejer vínculos de largo aliento con el territorio donde se despliega, como tampoco ha estado a la altura de construir conocimiento verdaderamente auténtico". (Saravia, en Tomassino, 2017:69)

Según Pablo Saravia (Tomasino, 2017), se trata de un conocimiento que tiene propiedades, historias y contenidos que se desarrollan en un contexto particular con características propias, que enuncian una forma de entender el entorno y sus procesos de cambio. Por lo tanto, funciona como una llave que abre una manera diferente de interpretar el territorio y sus actores. Esto último advierte sobre la profunda dimensión epistemológica que tiene este debate, ya que no solo se trata de la construcción de un conocimiento con otra $\square$ sonomía y actores, sino que tensiona su uso, origen y portadores.

Aspectos ya revisados, como el resguardo del patrimonio simbólico o cultural, la sostenibilidad social y política, asuntos asociados a saberes originarios del territorio, la cohesión, entre otros, son preocupaciones que toda universidad debe considerar al momento de decidir abrir una nueva sede o fortalecer los vínculos ya desarrollados.

Luego de recordar la hipótesis de este trabajo, acerca de que la legitimidad territorial de una universidad depende de un proceso complejo de compromisos, lealtades y afectos con su comunidad, cabe señalar que esta debe estar permanentemente informada de lo que la universidad hace con "sus" recursos naturales y con "su" gente en proyectos de investigación o de extensión y dar certeza (capital institucional, en el lenguaje de Boisier) de que estará de su lado al momento de certificar, por ejemplo, la instalación de una nueva fábrica o proyecto medioambiental, procesos que probablemente estarán institucionalizados desde el gobierno central.

Las siguientes discusiones, entonces, pretenden contribuir a generar un debate sobre las actitudes o conductas que una universidad debe establecer en su tarea de extensión/ vinculación. Para algunas universidades, abrir una sede en la actualidad muchas veces es señal de la oportunidad comercial que esa alternativa expansionista ofrece al directorio o financistas de las mismas más que de la real convicción de contribuir al desarrollo regio- 
nal/local. En el resto de Latinoamérica, la vinculación con la universidad pública comenzó mucho antes que en Chile.

Tal como señaló en párrafos anteriores, en Chile estamos un tanto desfasados en cuanto a la preocupación por la relación con el territorio. El tipo de reflexiones propiciadas en este artículo cuestiona si la identidad de la universidad con la localidad en la cual se inserta es un acto posterior que depende de la confianza que este centro de educación provoque en sus vecinos (o de cómo sean interpretado). En el otro polo aparecen centros de estudios insertos en malls o centros comerciales que se acercan más a los denominados "no lugares", 9 es decir, a espacios lineales, deslocalizados, sin identidad anclada. También está el caso de universidades públicas que se instalan en territorios desconocidos con inexistente vínculo con la gente y su historia. Algunos casos en Chile se constatan en la experiencia de la sede Melipilla de la Universidad de Valparaíso, que en 2006 abrió sus operaciones con magros resultados que la obligaron a cerrar al poco tiempo. O también el caso, en la misma comuna, de la Universidad del Pacífico, que apostó por la apertura de una sede en un territorio lejano a su casa central y desconocido, con ningún vínculo previo o natural, con una oferta programática distante de la tradición académica de su sello. Asimismo, recientemente el Estado chileno creó universidades públicas en dos regiones y se encuentra a punto de crear 16 Centros de Formación Técnicas (CFTs) en cada una de las regiones. En todos estos casos se debería haber realizado un estudio de las características demográficas, culturales y patrimoniales de los territorios.

A continuación, detallamos las discusiones cuya reflexión debería aportar a los tomadores de decisiones universitarias criterios de acción en sus políticas de extensión o vinculación con el medio.

\section{Discusión 1: Delimitación del territorio y alcance de la universidad. ¿Universidades globales, nacionales o locales?}

Según Castells, la globalización presenta una compleja superposición de redes globales y redes locales que tensionan las estrategias de vinculación de las empresas e instituciones. Las universidades no escapan a este escenario. Esta primera discusión se refiere a la necesidad que hoy tiene todo proyecto académico de declarar — con la ya abordada complejidad teórico-metodológica - la amplitud territorial o de alcance de sus acciones, también denominada su área de influencia territorial. En este sentido, las universidades deben responder a criterios de amplitud disciplinar o profesional de sus egresados. Insistimos en que deben precisar los límites geográficos y simbólicos de un cierto territorio donde desarrollará su proyecto. Esto permite generar responsabilidad social en su funcionamiento y credibilidad en la comunidad respecto de que "esa" universidad se comprometerá con la sostenibilidad de la comunidad y de su patrimonio.

Este aspecto va más allá de la extensión de los centros de práctica que tengan sus estudiantes o pasantías, o de los vínculos académicos o disciplinares de sus profesores; la

9) En el año 1992 Marc Augé introdujo la expresión "no lugar" en su libro Los no lugares, espacios del anonimato. Ha construido un bagaje bastante completo en torno al concepto de espacio en este sentido. La visión del lugar como objeto antropológico de intercambio, relación y encuentro, en cuanto a un opuesto que se configura en el escenario de la modernidad globalizada. 
cuestión radica en declarar el área de interés de esa universidad, hasta dónde le interesa cubrir el patrimonio socioambiental, el cuidado de sus aguas, el resguardo de petroglifos en la montaña, la formación de capital humano, etc. Como ya hemos señalado, hoy el concepto de "vinculación con el medio" resulta altamente impreciso, precario e insuficiente para fijar estándares de calidad en la relación universidad-comunidad. La ambigüedad del concepto "medio" no se compara con el de "territorio", que tiene un componente jurisdiccional anclado a definiciones antropológicas, identitarias y culturales, al llamado "imaginario urbano" de Lindon.

La evidencia hoy indica que en Chile algunos centros de educación superior, como también se mencionó, se instalan arbitrariamente incluso en espacios dentro de malls o centros comerciales, exacerbando el concepto de "no lugar" de Augé o de espacio sin identidad propia o local. Por ejemplo, tenemos el caso del empresario chileno Andronico Luksic, quien declaró a fines de 2018 que el primer centro en América Latina de la prestigiosa Universidad de Tsinghua, China, comenzaría a funcionar durante el próximo año en Chile. Esto, luego de que el Grupo Luksic firmara un convenio con la casa de estudios, que ya tiene tres otras sedes internacionales en Indonesia, Italia y Estados Unidos. Luksic señaló: "Estamos seguros que este será un nuevo polo académico del más alto nivel, destinado a impulsar el talento chileno, latinoamericano y chino en un mundo cada día más conectado y desafiante». ${ }^{10}$ Asimismo, sobre la alianza, comentó que "creemos que es una manera de colaborar con centros de conocimiento con las universidades locales (...) nos parece que es una manera de colaborar con Chile en un rubro tan fundamental como es el de la educación". En este ejemplo claramente no existe antecedente histórico que vincule a la universidad con su entorno, más bien se responde a intereses del capital del inversionista y a la lógica de intereses transnacionales en materia de formación que probablemente desconozcan las características territoriales del lugar donde se emplace este proyecto educativo.

\section{Discusión 2: Licenciamiento territorial (social) de la universidad. Nuevos desafíos}

De acuerdo con lo planteado por Boaventura de Sousa Santos, existen tres crisis en las universidades: la crisis de hegemonía, la de legitimidad y la institucional:

"La de legitimidad, fue provocada por el hecho de haber dejado de ser la universidad una institución consensual, frente a la contradicción entre la jerarquización de los saberes especializados de un lado, a través de las restricciones del acceso y certificación de las competencias, y de otro lado, por las exigencias sociales y políticas de la democratización de la universidad y la reivindicación de la igualdad de oportunidades para los hijos de las clases populares". (2007:22)

Al respecto, es conveniente preguntarse cómo una universidad asegura el cumplimiento de sus exigencias sociales y políticas de descentralización y cómo puede estar segura de que su proceso de relacionamiento comunitario considerará la aprobación de la comunidad cercana a las actividades del proyecto, sean estas académicas o de otra índole, tal

10) https://www.emol.com/noticias/Economia/2018/12/06/929934/Grupo-Luksic-firma-convenio-con-universidad-china-para-instalar-su-primera-sede-en-America-Latina.html 
como ocurre hoy con la aprobación e instalación de un proyecto minero, tecnológico o de energía, donde la empresa que entrega el servicio debe cumplir con una rigurosa normativa medioambiental que integre un proceso de consulta y participación ciudadana para garantizar cierta satisfacción de la comunidad frente a la responsabilidad en los procesos productivos que desarrolle la empresa.

Entendiendo y dejando en claro que estamos hablando de dimensiones societales de distinto tenor y calibre, el caso anterior debiera ser aplicable también a la educación superior. En contextos de gobernanza y de mayor control ciudadano es recomendable la participación de la ciudadanía en la definición e instalación de nuevas universidades o centros de investigación. Es más, podría existir algún consejo ciudadano que permita conocer las necesidades de la comunidad y su plan de desarrollo. Frente a la situación de cómo una Universidad decide instalarse en un territorio, más allá de la aprobación técnica del Ministerio de Educación, conviene preguntarse: ¿existe algún protocolo que exija a esa universidad la presentación de sus antecedentes u hoja de vida a la comunidad? Y, por otra parte: ¿dónde queda establecida la representatividad de la comunidad por parte de la universidad? Claramente, las respuestas a ambas preguntas sufren un rotundo no.

No existe en la legislación chilena alguna obligatoriedad en este sentido. Más bien, el único licenciamiento que se generaría si fuese así es de tipo de simbólico, es decir, en el concepto del chileno Sergio Boisier, se podría estar generando un acuerdo como parte del capital institucional del territorio, en base a intangibles como la confianza hacia esa universidad, pero nada desde el punto de vista jurídico. El licenciamiento se obtendría producto de negociaciones de la Universidad en el territorio, pero seguimos sosteniendo con ciertas dudas respecto a la espontaneidad, naturalidad y por supuesto calidad institucional de este proceso.

El peso curricular o biográfico en materia socio ambiental de cierta universidad o un incidente en el ámbito de la salud pública, son un elemento que queda solo en el plano de la justicia civil pero no es materia de esto que intentamos llamar "licenciamiento territorial". Al respecto es interesante analizar lo que sucedió con una Universidad privada en Chile. "En el año 2014 la Universidad del Pacífico firmó en febrero de 2010 un convenio con el Hospital San José de Melipilla para usarlo como campo clínico para sus alumnos de Enfermería por los siguientes 15 años. Así, sin siquiera tener aún la carrera acreditada, la universidad privada "amarró" su presencia hasta 2025 en un hospital público (Ciper Chile, 2014). Fue en el marco de esta relación universidad- hospital que una estudiante en práctica de la carrera de enfermería, aplicó equivocadamente un procedimiento médico que le significó la vida a dos personas adultos mayores.

Esta situación creó un compleja y tensa situación, pero la universidad siguió funcionando en la comuna, hasta 2018, donde por razones de gestión fue intervenida por la autoridad ministerial terminando abruptamente su proyecto. Este es un caso aislado e involuntario, qué duda cabe, pero cuya repercusión mediática dañó irreversiblemente la calidad del proyecto educativo de esta casa de estudios. Quizás si hubiese existido un canal de comunicación previo entre la comunidad y la Universidad hubiese permitido un mayor control sobre la calidad del proyecto educativo, el error técnico en el procedimiento de la estudiante hubiese sido abordado de mejor forma y los estudiantes involucrados podrían haber conocido el tipo de enfermedades predominantes de la población comunal, sus creencias al respecto, los tipos de relaciones familiares, etc. Otro punto que debe considerarse es el nivel de cre- 
dibilidad que una universidad tenga en la comunidad respecto de su valoración de los principios medioambientales. Que una universidad registre en su historial el respaldo científico o técnico hacia una actividad extractiva por ejemplo que luego generó daños importantes al patrimonio natural del territorio, es sin dudas un problema grave y que debiera afectar la continuidad de una eventual licencia.

\section{Discusión 3: Incorporación de las universidades en las estrategias regionales/ comunales de desarrollo}

Este punto es quizás la mejor evidencia o forma de constatar el real compromiso de una universidad con el desarrollo local. Se refiere a discutir si la universidad es considerada efectivamente por la autoridad política en la definición de estrategias públicas de desarrollo. Por ejemplo, a nivel regional. Las estrategias regionales de desarrollo en Chile (ERD) son instrumentos públicos de planificación regional que convocan a distintos actores clave del desarrollo. La invitación a participar o no evidencia el alcance territorial de una determinada universidad, supone la visibilidad que esa institución académica tiene para el desarrollo de ese territorio.

La nueva institucionalidad del reciente Ministerio de Ciencia y Cultura establece como medida esencial la creación de un Sistema Regional de Gestión del Conocimiento que incluirá el fortalecimiento del vínculo educación superior de regiones con su entorno regional. Este sistema buscará promover en las instituciones de educación de regiones su tránsito desde el modelo tradicional "centrado en lo interno" hacia un paradigma de institución interactiva, capaz de revalorizar el espacio local y regional del desarrollo, construir conocimiento desde y para los territorios, en un proceso horizontal y bidireccional de mutuo beneficio con los actores públicos, privados y sociales del respectivo entorno regional y fortalecer un ambiente de confianzas recíprocas y rendición de cuentas, e introducir incentivos correctivos en materia de donaciones, que favorezcan a las instituciones de Educación Superior Regionales (Comisión Asesora Presidencial en Descentralización y Desarrollo Regional, 2014).

Para el desarrollo de los territorios resulta esencial la formación de personas con conciencia regional derivada del conocimiento profundo de la región, su historia, sus potencialidades y valoración como espacio para la creación de futuro. Hemos perdido, y por lo tanto es importante recuperar, la formación de educación cívica en los niveles de educación básica y media, mientras que en los niveles de educación superior se requiere de políticas que incorporen, motiven y diversifiquen formación con pertinencia territorial al servicio del desarrollo endógeno (Comisión Asesora Presidencial en Descentralización y Desarrollo Regional, 2014). Por ello se propone incorporar al sistema de educación regional un enfoque endógeno y territorial del desarrollo con pertinencia y contenidos específicos propios de cada región y de vinculación con el respectivo entorno en los procesos de formación del capital humano (por ejemplo, incorporar contenidos de pueblos originarios, memorias históricas), además de promover el diseño y las renovaciones curriculares pertinentes al entorno local y regional en el sistema de educación técnica y universitaria de pre y posgrado, considerando elementos del entorno en la oferta formativa de cursos electivos, prácticas intermedias y profesionales y proyectos de título y tesis de grado.

También se propone generar un órgano regional descentralizado, con dependencia administrativa del GORE y con representación público-privada-académica y étnica (en este 
último caso según corresponda) en materia de ciencia, tecnología e innovación, acorde a los desafíos de desarrollo de cada región, dotándolo de los instrumentos adecuados, en concordancia con las actuales herramientas de planificación territorial (políticas regionales de ciencia, tecnología e innovación, y estrategias regionales de innovación preexistentes) y con sujeción a los instrumentos de planificación regional del GORE (Estrategia Regional de Desarrollo, Políticas Regionales, futuro Plan Regional de Ordenamiento Territorial).

La nueva institucionalidad considera el otorgamiento de incentivos regionales y nacionales para que el sistema universitario estatal regional cree Centros de Pensamiento Estratégico Territorial. La creación del Fondo antes descrito será complementada por un sistema de incentivos especialmente destinados a que las universidades estatales regionales cumplan con su rol de vinculación con el medio focalizando su accionar en la generación de estos bienes públicos. Es decir, se trata de poner a disposición de estas entidades mecanismos para hacer efectivo su aporte a las políticas públicas regionales y, en especial, para generar conocimiento pertinente a las vocaciones y desafíos del desarrollo de cada región, a la vez que contribuir a la formación de cuadros profesionales y líderes comprometidos con el territorio y servir de espacio de articulación de los actores del desarrollo regional.

Sin embargo, al respecto la nueva Ley de Educación Superior establece la consideración de universidades solo públicas o del $\mathrm{CRUCH}$ y que además cumplan con el requisito de tener ciertos años mínimos de acreditación; solo ellas podrán aportar a la definición de estrategias de desarrollo. Esto nos lleva a pensar cómo se permitirá la participación de las demás universidades - que no cumplen con los requisitos planteados-, que quedarán excluidas de alguna posibilidad de aportar planificadamente al desarrollo del territorio donde se insertan sus actividades. ¿Solo podrán realizar actividades individuales y autónomas descontextualizadas de un plan concertado de desarrollo? La nueva institucionalidad margina de cierta forma a las universidades privadas o no adscritas al CRUCH en esta misión.

\section{Discusión 4: Colaboración versus competencia en el territorio}

Como se señaló, la tercera crisis de la universidad según Boaventura de Sousa Santos, fue la institucional, resultado de la contradicción entre la reivindicación de la autonomía en la definición de valores y objetivos de la universidad y la presión creciente para someterla a criterios de la eficiencia y la productividad de naturaleza empresarial o de responsabilidad social. Esta productividad muchas veces requiere la competencia entre instituciones. Señala González:

"La competencia, como factor que cruza transversalmente a las universidades chilenas de todo tipo, han generado que, a pesar del reconocimiento de esas raíces históricas comunes, todas las universidades asuman un modo de comprender y publicitar su funcionalidad hacia los territorios, altamente heterogéneo, funcional a los recursos disponibles, que hace que el mapeo de experiencias tenga forma de una gran diáspora o explosión de sentidos y prácticas". (2017:121)

Esta diversidad de proyectos educativos, con ideales distintos, valores diferentes, estrategias de vinculación distintas, sumada a las exigencias tecnocráticas del Estado por evidenciar su aporte sustantivo al "medio" y la fuerte competencia por los recursos públicos provoca cierto grado de conflictividad y afecta el desarrollo armónico de la región. El norte 
debería estar dado por la posibilidad colaborativa entre las universidades en función del bien común del territorio. La asociatividad inter universidad y con las estructuras de las propias comunidades tendría que ser un factor presente en la medición de vinculación con el medio, independiente de sus definiciones religiosas o ideológicas.

\section{Consideraciones finales}

Creemos que la instalación de esta nueva mirada es un paso importante para que los decidores de inversiones en proyectos universitarios adopten decisiones estratégicas de efectivo relacionamiento con el territorio. En nuevos contextos de gobernanza y sostenibilidad, esta condición resulta sinequanon para el desarrollo. Por otra parte, debe precisarse en la medición de la vinculación con el medio el concepto de territorio en reemplazo de otros familiares o que históricamente han sido asociados, como entorno, medio, área de influencia, etc., que revisten mayor ambigüedad conceptual. Avanzar en una definición que utilice conceptos como identidad, discursos, multiculturalidad, desarrollo local, entre otros, habla más de un enfoque territorial en las futuras definiciones de vinculación con el medio.

Finalmente, para una mejor definición sobre relaciones de una carrera o programa de estudio deben construirse indicadores ad hoc que reemplacen o complementen los relacionados con aportes en el ámbito disciplinario o profesional sin hacer alusión al territorio donde se insertan sus actividades. $Y$ creemos que, junto con precisarlo, debe realizarse un esfuerzo por establecer indicadores de vinculación territorial, tales como si la universidad determinada forma parte del consejo de las tierras de la comunidad, de las mesas de inversión comunal, de los consejos de la sociedad civil del municipio, o si es considerada activamente en el Plan de Desarrollo Comunal o Regional, etc., que evidencie ese compromiso claro con el desarrollo del entorno geográfico y cultural del lugar y no necesariamente disciplinar y/o profesional, como se hace en la actualidad.

El acento debe ponerse con claridad en la definición territorial de la vinculación con el medio. Más allá del planteamiento de si la vinculación con el medio transita entre un extensionismo difusionista y la extensión crítica (en el lenguaje de González), es fundamental conocer y valorar las características territoriales en su opción de vinculación con el medio. Frente a cualquier política expansionista de una universidad, esta ya no solo deberá sopesar variables economicistas en su decisión. El territorio y su complejidad así lo exigen en una justa convivencia.

\section{Referencias bibliográficas}

Arzeno, M. (2018). Extensión en el territorio y territorio en la extensión. Aportes a la discusión desde el campo de la Geografía. +E: Revista de Extensión Universitaria, 8(8), 3-11. Santa Fe, Argentina: Ediciones UNL. doi: 10.14409/. v8i8.Ene-jun.7709

Boisier, S. (1999).Teorías y metáforas sobre el desarrollo Territorial. Santiago de Chile: CEPAL.

CNA (2014). Resolución Exenta DJ N 001-4. Aprueba Reglamento sobre Área de Acreditación. Recuperada de: https://www.cnachile.cl/docs/transparencia/res\%2001-13.pdf (2016). Guía para la Autoevaluación Interna. Acreditación Institucional Universidades.

Comisión Asesora Presidencial en Descentralización y Desarrollo Regional (2014). Propuesta de Política de 
Estado y Agenda para la Descentralización y el Desarrollo Territorial de Chile. Hacia un país desarrollado y justo. 7 de octubre de 2014.

Garretón, M. (2009). Matriz Sociopolítica y Desarrollo Socioeconómico en Chile. Diplomacia, Estrategia y Política, (9). Recuperado de: http://www.manuelantoniogarreton.cl/documentos/artespanolmatrizippg.pdf

González López, B. (2017). Extensión universitaria en Chile: discursos y prácticas sobrevivientes. +E Revista de Extensión Universitaria, 7(7), 110-121. Santa Fe, Argentina: Ediciones UNL.

Llanos Hernández, L. (2010). El concepto del territorio y la investigación en las ciencias sociales. Agricultura, sociedad y desarrollo, 7(3). Universidad Autónoma Chapingo, México.

Lindon, A. (2007). La ciudad y la vida urbana a través de los imaginarios urbanos. EURE, XXXIII(99), 7-16. Santiago de Chile.

Menardi, M.; Tecco, A. y López, S. (2016). El rol de las Universidades en la Generación de capacidades Territoriales para la Innovación y el Desarrollo. Revista de Administración Publica y Sociedad.

Méndez Sastoque, M. \& Jurado, G. (2016). La universidad en la promoción de procesos de desarrollo local: reflexiones alrededor del proyecto "Universidad en el Campo". Pampa (Santa Fe), (13), 115-126.

Nogué, J. y Vela, J. (2011). La dimensión comunicativa del paisaje. Una propuesta teórica y aplicada. Revista de Geografía Norte Grande, 49, 25-43.

OCDE (2017). Evaluaciones de Políticas Nacionales de Educación. Educación en Chile.

Quinteros, C. (2018). Competencias Profesionales del Trabajador Social para la Gestión Territorial: Aproximación exploratoria desde los planes de estudio de Pre Grado. Interacción y Perspectiva. Revista de Trabajo Social, 8(1), 11-29.

Rivera, F.; Rivera-Vargas, P.; Alonso, C. (2018). Una mirada territorial al sistema universitario chileno. El caso de la Universidad Regional de Aysén (UAY). Estudios Pedagógicos, XLIV(1), 427-443.

Sousa Santos, B. de (2007). La Universidad en el siglo XXI. Para una reforma democrática y emancipatoria de la universidad. Colección Universidad.

Tommasino, H. (2017). Vinculación con el medio y territorio. Heterogeneidad de modelos, prácticas y sentidos en las universidades chilenas. Observatorio de Participación Social y Territorio. Universidad de Playa Ancha, Chile. Universidad de Santiago de Chile (USACH) (2010). 161 años de historia. Recuperado de: https://www.usach. cl/sites/default/files/documentos/files/revistausach-bicentenario-n1_0.pdf

Universidad Alberto Hurtado (2018). Política de Vinculación con el Medio. Recuperado de: http://mailing. uahurtado.cl/2018/comunicaciones_rectoria/marzo/pdf/politica_vinculacion_con_el_medio.pdf

Von Baer, H. (2006). Descentralización y Desarrollo Local/Regional: La Tarea Pendiente de Modelo "Chilensis" de Desarrollo Propuestas para un estilo de desarrollo armónico y sustentable, social y territorialmente integrado, con participación de la sociedad civil organizada. Agenda Pública, V(10). Recuperado de: http://www. agendapublica.uchile.cl/n10/baer.pdf

\section{Leyes}

Ley N²1.091 Sobre Educación Superior. (2018) 